\title{
Adverse childhood experience detrimental to health and function.
}

\author{
Trevor Archer* \\ Department of Psychology, Gothenburg University, Gothenburg, Sweden
}

Accepted on November 01, 2017

Traumatization, the operative infliction of a trauma/ traumatic event, may be described as the process(es) through which the criteria of posttraumatic stress disorder (PTSD) are achieved. Trauma or a traumatic event stems from an overwhelming/excruciating bombardment of extreme stress that exceeds individuals' ability to cope, or integrate the cognitive-climate involved with that or those experience(s). Psychologically traumatic experiences often involve physical trauma that threatens individuals' survival and sense of security with highly detrimental consequences for brain and CNS development [1]. In a group of childhood trauma survivors of foster care settings, trait anger, anger rumination, emotional abuse, and PTSD re-experiencing symptoms were expressed repeatedly with anger rumination related to meaningful past events as the central factor [2]. Tonic immobility, a temporary state of motor inhibition which is elicited in the context of inescapable threat and perception of entrapment, presents an involuntary motor and vocal inhibition reaction, considered the last-ditch response of the defensive cascade model. PTSD patients with high self-reported tonic immobility may offer a distinct stress response pattern, i.e., a proneness or predisposition to be afflicted by immobility. It presents a state of physical immobility associated with extreme stress and the development of PTSD and peritraumatic tonic immobility renders a massive link to sexual trauma, especially in child sufferers but strongly too in adolescents and adults, than to other types of trauma experience in the general population [3]. In a group of women $(n=46)$ exposed to unwanted childhood sexual contact, negative beliefs about the self independently mediated the relation between peritraumatic perceptions of inescapability and PTSD [4]. Among a population of young, healthy adults selected for childhood abuse-threat, and in comparison with an un-abused control group, it was observed that there was less gray matter in the female participants within the visual posterior precuneal region following both subtypes, abuse-threat and deprivation, of childhood abuse than controls whereas the male participants showed a lesser volume of gray matter in the postcentral gyrus after childhood deprivation compared with abuse [5]. Childhood trauma exposure as flooding, earthquakes and rainstorms victims in China exerted a direct and positive association to depressiveness and PTSD arising from sleep problems, social support and resilience, fear and ruminations, self-esteem and hope and negative cognitions [6-11].

Among a group of Palestinian children, it was observed that that $43 \%$ of those participating reported playing or visiting a nice place as earliest memories, and about one third (30\%) of the traumatic events or accidents $(30 \%)$ or miscellaneous events $(27 \%)$ linked to the memories [12]. The individual and social orientations of these children were almost equally common, the emotional tone mainly neutral (45.5\%), with $60 \%$ remembering a specific event/episode. Furthermore, the boys remembered a greater number of earliest memories involving traumatic events or accidents, whereas girls remembered more social events. Finally, the trauma of war was associated with reduced positive emotional tone and with greater specificity of these memories. Childhood trauma has been found to provide the origin for multiple expressions of psychopathology [13-15] whereas the reverse holds true even if there is a lack of awareness [16] i.e. higher recalled levels of benevolent childhood experiences predicted less PTSD symptoms and stressful life events together with much fewer adverse life events. A powerful relationship between 'negative affect interference' and hedonic deficit, on the one hand, and, on the other hand, expressions of anhedonia, positive-negative affect regulation problems, negative affect, and presence of neuroticism [17]. Childhood traumatic experiences are associated both with exacerbated negative affect liability, serious clinical depression, schizophrenic and psychotic episodes and bran responding to negative stimuli, i.e. negative versus positive facial expressions [18-21]. Certain evidence appears to imply that prior to and during the point of 'transition'; affective symptoms contribute the mediatory pathway bridging the transition from sexual trauma to prodromal and full-fledged psychosis [22]. It has been shown also that maladaptive/self-destructive cognitive behavior, negative cognitive and coping styles mediate associations between childhood trauma and neuropsychiatric consequences [23,24]. Rumination, the tendency to fixate constantly upon negative emotions/feelings and continually think about (ruminate) upon causation, implications, coping failures and ramifications, often with childhood or adolescent emergence has been implicated as an underlying mediator of affective disorders [25-27]. In a study of the mediatory role of rumination as the connecting association between childhood trauma and anxiety-depressive mood states, including affective liability, 207 participants (non-clinical) responded to the childhood questionnaire, the Ruminative Response Scale, the Beck Depression Inventory, The State Anxiety Inventory and the Affective Liability Scale [28]. The authors observed 
that rumination presented a notable mediatory association between childhood trauma and anxiety-depression with a direct relationship between the different mood states and rumination that was most pronounced among the female participants [29].

Childhood trauma bestows manifold risks driving the maladjusted neurodevelopment of psychiatric disorders in multifarious domains. The prevailing notion that adverse childhood experience, excessive normal experience, is induced through an excess of stress hormones upon synaptic formation and neuronal pruning such that neurodevelopmental pathways are disrupted with consequential structural and functional predispositions to the pathophysiology of brain disorders [30-32]. Adult participants presenting childhood trauma displayed reduced regional homogeneity in the bilateral superior temporal gyrus and insula, and the right inferior parietal lobule, together with elevated regional homogeneity in the right cerebellum and left middle temporal gyrus that was associated with disturbances of intrinsic brain function, and in particular dysregulation of the default mode network [33]. Hippocampal, anterior cingulate cortex and/or prefrontal cortical dysfunction due to hypothalamic-pituitaryadrenaline (HPA) abnormalities elicited by emotional and stress upheaval following childhood trauma and/maltreatment has been observed also [34-37]. Taken together, thereis available a plethora of evidence that childhood adverse experience is linked to later brain structural abnormality, neuropsychiatric expression and epigenetic-neurochemical biomarker dysregulation [38-41]. Thus, the anterior cingulate cortex volume was reduced markedly among the PTSD participants in comparison with healthy controls [36]. In a group of 47 schizophrenic or schizoaffective patients it was found that the total Childhood Trauma Questionnaire scores were associated positively with the activation of the posterior cingulate cortex/precuneus with another positive association between the childhood trauma exposure incidents and the activation of the dorsomedial prefrontal cortex with a negative association with activation between of the anterior section of the tempero-parietal junction and trauma [42], thereby disclosing functional alterations in a Theory-of-Mind task.

The emotional Stroop task, measuring participants' response times to name colours of negative words, induces an effect involving the attention and slow response times elicited by words with 'emotional-relevance' to the individual tested thereby offering estimations of clinically-convictive words and associations, particularly in the context of PTSD $[43,44]$. Further, it has been registered that exposure peer verbal abuse during the individual's childhood years induces an aversive stimulus that is later associated with meaningful functional changes within the emotional regulation network, by the expression of a hypersensitivity/oversensitivity to swear words, during middle adolescence with concomitant functional alterations to the ventrolateral prefrontal cortex [45]. Female participants, aged 23 to 30 years, presenting a background history of childhood abuse $(n=15)$, or no abuse $(n=17)$, displayed reduced engagement of the frontal-parietal regions appertaining to cognitive control mechanisms and the higher level of engagement of a ventral attention-surveillance network during presentations (blocks) of both, clinical and non-clinical versions of the Emotional Stroop task [46]. Furthermore, these participants exhibited a lower level of suppression of those brain regions involved in self-referential processes, the interpretation of incoming information in relation to oneself through applications of one's self-concept as a background template for the new information, for threat blocks, but higher levels of suppression of these regions for the positive blocks. The severity of displayed avoidance symptoms was linked to the sustained activation of the lateral prefrontal regions, while, contrastingly, hyperarousal/reexperiencing symptoms were linked to the sustained activity of temporal regions. In a population of automobile accident sufferers ( $n=194,36.64 \pm 12.3$ years), the pictorial version of the Emotional Stroop task was applied [47]. They authors obtained marked and specific elevations of reaction times associated with the presentation of trauma-related material with an age effect by which the older group failed to display the reaction time increase. Negative cognitions and mood instability following PTSD may predict eventual alcohol and other substance abuse disorders [48].

In conclusion, childhood adverse environments and/ or trauma and abuse develops invariably into a wide range of maladaptive behaviors that may be identified through their negative cognitive-emotional consequences whether or not the individual afflicted present clinical or prodromal diagnoses extending from substance abuse and clinical depression, borderline personality to psychosis and schizophrenia spectrum disorders. Movement disorders, including peritraumatic immobility and involuntary dyskinesia, cognitive expressions, such as rumination, negative cognitions, and word recall, and emotional features involving the stroop emotional effect, mood instability and anxiety-depressive affective state. Regional structuralvolumetric and functional alterations extend over prefrontal areas, involving dorsolateral, dorsomedial and ventrolateral projections, anterior and posterior cingulate cortex, frontoparietal regions and the temperoparietal junctions following childhood maltreatment and traumatic experience; an interference to normal development that is far-reaching, far-ranging and permanent in its destruction and consequence.

\section{References}

1. Ganapathi R, Manda K. Later life changes in hippocampal neurogenesis and behavioral functions after low-dose prenatal irradiation at early organogenesis stage. Int $\mathrm{J}$ Radiat Oncol Biol Phys. 2017;98(1):63-74.

2. Glück TM, Knefel M, Lueger-Schuster B. A network analysis of anger, shame, proposed ICD-11 post-traumatic stress disorder, and different types of childhood trauma in foster care settings in a sample of adult survivors. Eur J Psychotraumatol. 2017;8:1372543. 
3. Kalaf J, Coutinho ESF, Vilete LMP, et al. Sexual trauma is more strongly associated with tonic immobility than other types of trauma - A population based study. J Affect Disord. 2017;215:71-6.

4. Van Buren BR, Weierich MR. Peri-traumatic tonic immobility and trauma-related symptoms in adult survivors of childhood sexual abuse: The role of post-trauma cognitions. J Child Sex Abus. 2015;24(8):959-74.

5. Everaerd D, Klumpers F, Zwiers M, et al. Childhood abuse and deprivation are associated with distinct sex-dependent differences in brain morphology. Neuropsychopharmacology. 2016;41(7):1716-23.

6. Quan L, Zhen R, Yao B, et al. The role of perceived severity of disaster, rumination and trait resilience in the relationship between rainstorm-related experiences and PTSD amongst Chinese adolescents following rainstorm disasters. Arch Psychiatr Nurs. 2017;31(5):507-15.

7. Zhen R, Quan L, Yao B, et al. Understanding the relationship between rainstorm-related experiences and PTSD among Chinese adolescents after rainstorm disaster: The roles of rumination and social support. Front Psychol. 2016;7:1407.

8. Zhen R, Quan L, Zhou X. Fear, negative cognition and depression mediate the relationship between traumatic exposure and sleep problems among flood victims in China. Psychol Trauma. 2017.

9. Zhou X, Wu X, Li X, et al. The role of posttraumatic fear and social support in the relationship between trauma severity and posttraumatic growth among adolescent survivors of the Yaan earthquake. 2016.

10. Zhou X, Wu X, Zhen R. Understanding the relationship between social support and posttraumatic stress disorder/ posttraumatic growth among adolescents after Ya'an earthquake: The role of emotion regulation. Psychol Trauma. 2017a;9(2):214-21.

11. Zhou X, Wu X, Zhen R. Self-esteem and hope mediate the relations between social support and post-traumatic stress disorder and growth in adolescents following the Ya'an earthquake. Anxiety Stress Coping. 2017b;1-14.

12. Peltonen K, Kangaslampi S, Qouta S, et al. Trauma and autobiographical memory: Contents and determinants of earliest memories among war-affected Palestinian children. Memory. 2017;1-11.

13. Asselmann E, Stender J, Grabe HJ, et al. Assessing the interplay of childhood adversities with more recent stressful life events and conditions in predicting panic pathology among adults from the general population. $\mathrm{J}$ Affect Disord. 2018;225:715-22.

14. Kilicaslan EE, Esen AT, Kasal MI, et al. Childhood trauma, depression and sleep quality and their association with psychotic symptoms and suicidality in schizophrenia. Psychiatry Res. 2017;1781(17):30902-12.
15. Newbury JB, Arseneault L, Moffitt TE, et al. Measuring childhood maltreatment to predict early-adult psychopathology: Comparison of prospective informantreports and retrospective self-reports. J Psychiatr Res. 2017;96:57-64.

16. Narayan AJ, Rivera LM, Bernstein RE, et al. Positive childhood experiences predict less psychopathology and stress in pregnant women with childhood adversity: A pilot study of the benevolent childhood experiences (BCEs) scale. Child Abuse Negl. 2017;2134(17):303587.

17. DePierro J, D'Andrea W, Frewen P, et al. Alterations in positive affect: Relationship to symptoms, traumatic experiences and affect ratings. Psychol Trauma. 2017.

18. Aas M, Dieset I, Hope S, et al. Childhood maltreatment severity is associated with elevated C-reactive protein and body mass index in adults with schizophrenia and bipolar diagnoses. Brain Behav Immun. 2017a;65:342-9.

19. Aas M, Henry C, Bellivier F, et al. Affective lability mediates the association between childhood trauma and suicide attempts, mixed episodes and co-morbid anxiety disorders in bipolar disorders. Psychol Med. 2017b;47(5):902-12.

20. Aas M, Kauppi K, Brandt CL, et al. Childhood trauma is associated with increased brain responses to emotionally negative as compared with positive faces in patients with psychotic disorders. Psychol Med. 2017c;47(4):669-79.

21. Church C, Andreassen OA, Lorentzen S, et al. Childhood trauma and minimization/denial in people with and without a severe mental disorder. Front Psychol. 2017;8:1276.

22. Thompson A, Marwaha S, Nelson B, et al. Do affective or dissociative symptoms mediate the association between childhood sexual trauma and transition to psychosis in an ultra-high risk cohort? Psychiatry Res. 2016;236:182-5.

23. Kanehisa M, Kawashima C, Nakanishi M, et al. Gender differences in automatic thoughts and cortisol and alphaamylase responses to acute psychosocial stress in patients with obsessive-compulsive personality disorder. J Affect Disord. 2017;217:1-7.

24. Ninomiya T, Oshita H, Kawano Y, et al. Reduced white matter integrity in borderline personality disorder: A diffusion tensor imaging study. J Affect Disord. 2018;225:723-32.

25. Alioto A, Di Lorenzo C. Long-term follow-up of adolescents treated for rumination syndrome in an inpatient setting. J Pediatr Gastroenterol Nutr. 2017.

26. Malfliet A, Van Oosterwijck J, Meeus M, et al. Kinesiophobia and maladaptive coping strategies prevent improvements in pain catastrophizing following pain neuroscience education in fibromyalgia/chronic fatigue syndrome: An explorative study. Physiother Theory Pract. 2017;33(8):653-60. 
27. Sellers R, Wells A, Morrison AP. Are experiences of psychosis associated with unhelpful metacognitive coping strategies? A systematic review of the evidence. Clin Psychol Psychother. 2017.

28. Kim JS, Jin MJ, Jung W, et al. Rumination as a mediator between childhood trauma and adulthood depression/ anxiety in non-clinical participants. Front Psychol. 2017;8:1597.

29. Absah I, Rishi A, Talley NJ, et al. Rumination syndrome: Pathophysiology, diagnosis and treatment. Neurogastroenterol Motil. 2017;29(4).

30. Berens AE, Jensen SKG, Nelson CA. Biological embedding of childhood adversity: From physiological mechanisms to clinical implications. BMC Med. 2017;15(1):135.

31. Lawson GM, Camins JS, Wisse L, et al. Childhood socioeconomic status and childhood maltreatment: Distinct associations with brain structure. PLoS ONE. 2017;12(4):e0175690.

32. Raymond C, Marin MF, Majeur D, et al. Early child adversity and psychopathology in adulthood: HPA axis and cognitive dysregulations as potential mechanisms. Prog Neuropsychopharmacol Biol Psychiatry. 2017;5846(17):30098-2.

33. Lu S, Gao W, Wei Z, et al. Intrinsic brain abnormalities in young healthy adults with childhood trauma: A restingstate functional magnetic resonance imaging study of regional homogeneity and functional connectivity. Aust N Z J Psychiatry. 2017;51(6):614-23.

34. Bruenig D, Morris CP, Mehta D, et al. Nitric oxide pathway genes (NOS1AP and NOS1) is involved in PTSD severity, depression, anxiety, stress and resilience. Gene. 2017;625:42-48.

35. Goodman J, McIntyre CK. Impaired spatial memory and enhanced habit memory in a rat model of post-traumatic stress disorder. Front Pharmacol. 2017;8:663.

36. Rinne-Albers MA, Pannekoek JN, van Hoof MJ, et al. Anterior cingulate cortex grey matter volume abnormalities in adolescents with PTSD after childhood sexual abuse. Eur Neuropsychopharmacol. 2017;977X(17):30899-4.

37. Shoshan N, Akirav I. The effects of cannabinoid receptors activation and glucocorticoid receptors deactivation in the amygdala and hippocampus on the consolidation of a traumatic event. Neurobiol Learn Mem. 2017;144:24858.

38. Feifel AJ, Shair HN, Schmauss C. Lasting effects of early life stress in mice: Interaction of maternal environment and infant genes. Genes Brain Behav. 2017.

39. Frodl T, Janowitz D, Schmaal L, et al. Childhood adversity impacts on brain subcortical structures relevant to depression. J Psychiatr Res. 2017;86:58-65.
40. Moran JK, Crombach A, Elbert T, et al. The individual contribution of DSM 5 symptom clusters of PTSD, life events and childhood adversity to frontal oscillatory brain asymmetry in a large sample of active combatants. Biol Psychol. 2017;129:305-13.

41. Smeijers D, Bulten E, Franke B, et al. Associations of multiple trauma types and MAOA with severe aggressive behavior and MAOA effects on training outcome. Eur Neuropsychopharmacol. 2017;977X(17):30293-6.

42. Quidé Y, Ong XH, Mohnke S, et al. Childhood trauma-related alterations in brain function during a theory-of-mind task in schizophrenia. Schizophr Res. 2017;9964(17):30083-X.

43. Khanna MM, Badura-Brack AS, McDermott TJ, et al. Veterans with post-traumatic stress disorder exhibit altered emotional processing and attentional control during an emotional Stroop task. Psychol Med. 2017;47(11):201727.

44. Wittekind CE, Muhtz C, Moritz S, et al. Performance in a blocked versus randomized emotional Stroop task in an aged, early traumatized group with and without posttraumatic stress symptoms. J Behav Ther Exp Psychiatry. 2017;54:35-43.

45. Lee SW, Choi J, Lee JS, et al. Altered functions of ventrolateral prefrontal cortex in adolescents with peer verbal abuse history. Psychiatry Investig. 2017;14(4):44151.

46. Mackiewicz Seghete KL, Kaiser RH, DePrince AP, et al. General and emotion-specific alterations to cognitive control in women with a history of childhood abuse. Neuroimage Clin. 2017;16:151-64.

47. Bielecki M, Popiel A, Zawadzki B, et al. Age As moderator of emotional stroop task performance in posttraumatic stress disorder (PTSD). Front Psychol. 2017;8:1614.

48. Boland M, Rielage JK, Hoyt T. The power of negative mood in predicting posttraumatic stress disorder and alcohol abuse comorbidity. Psychol Trauma. 2017.

\section{*Correspondence to:}

Trevor Archer

Department of Psychology

Gothenburg University

Box 500, SE-405 30 Gothenburg

Sweden

Tel: +46 317864694

E-mail: trevor.archer@psy.gu.se 